\title{
Dosimetric Evaluation of the Physical Parameters for Different Energies in Advanced Radiotherapy Technique for Liver Cancer
}

\author{
S. Hassn ${ }^{1}$, Khaled M. El-Shahat ${ }^{2}$, M.F.Eissa ${ }^{3}$ and A.H. Aly ${ }^{3}$ \\ ${ }^{(1)}$ Minia Oncology Center, Ministry of Health and Population, Minia, Egypt \\ ${ }^{(2)}$ Clinical Oncology Department, Faculty of Medicine, Al-Azhar University, Egypt \\ ${ }^{(3)}$ Physics Department, Faculty of Sciences, Beni-Suef University, Beni Suef, Egypt
}

\begin{tabular}{|c|c|}
\hline ARTICLE INFO & ABSTRACT \\
\hline Article history: & \multirow{7}{*}{$\begin{array}{l}\text { This work aims to study dosimetrically compared } 6 \mathrm{MV}, 10 \mathrm{MV} \text {, and dual energies (DE) } \\
\text { photon beam energies in patients with liver cancer. Evaluating the effect of using } \\
\text { different energies on intensity-modulated radiation (IMRT) outcome were selected. Step- } \\
\text { and-shoot IMRT treatment plans were designed for delivery on an Elekta linear } \\
\text { accelerator with } 160 \text { leaves. Identical optimization constraints were applied for all energy } \\
\text { plans. Parameters such as beam angle and number of beams were kept constant to } \\
\text { achieve the same clinical objectives. Both qualitative and quantitative methods were used. } \\
\text { Many physical indices for Planning Target Volume (PTV), the relevant Organs at Risk } \\
\text { (OARs) as mean dose (Dmean), maximum dose (Dmax), 95\% dose (D95), and also the } \\
\text { number of monitor units (MU) were applied. }\end{array}$} \\
\hline Received: 31 ${ }^{\text {st } J a n . ~} 2021$ & \\
\hline Accepted: $5^{\text {th }}$ Aug. 2021. & \\
\hline \multirow{6}{*}{$\begin{array}{l}\text { Keywords: } \\
\text { 6-MV, 10-MV, } \\
\text { Double Photon Energies; } \\
\text { Intensity-Modulated Radiation } \\
\text { Therapy (IMRT) photon } \\
\text { energy. }\end{array}$} & \\
\hline & \\
\hline & \\
\hline & \\
\hline & $\begin{array}{l}\text { Twenty eight virtual IMRT treatment plans were involved in this study. the plans } \\
\text { depended on Monaco's (IMRT) treatment plan outcome. For each case, three plans with } \\
\text { the same beam geometry were created using } 6 \mathrm{MV}, 10 \mathrm{MV} \text {, and DE. For dual energy } \\
\text { plans, all cases were optimized with identical planning objectives and normalized such } \\
\text { that } 98 \% \text { of the target received } 100 \% \text { of the prescription dose. }\end{array}$ \\
\hline & $\begin{array}{l}\text { The three techniques showed comparable PTV inhomogeneity and conformity for all } \\
\text { patient's differences within the median values }<0.6 \% 10 \mathrm{MV} \text { and DE plans and } \\
\text { a statistically important reduction in the total number of monitor units (MU) of } 14.2 \% \\
\text { (p<0.01) and } 13.3 \% \text { (p }<0.01 \text { ) as compared to } 6 \mathrm{MV} \text {, respectively. It could be concluded } \\
\text { that each dual energy and } 10 \mathrm{MV} \text { energy had similar PTV dosimetry characteristics. }\end{array}$ \\
\hline
\end{tabular}

\section{INTRODUCTION:}

Radiation therapy uses targeted radiation for the treatment of cancer. The radiation is often in form of $\mathrm{x}$-ray beams. Conventional external beam radiation therapy is not usually used for secondary liver cancer; however, two specialized forms of radiotherapy may be used in some cases [1].

In the treatment of hepatocellular carcinoma (HCC), radiotherapy (RT) had a limited role because the whole liver is little tolerant to RT in addition to the risk of radiation-induced liver disease (RILD) [2]. Even though in many guidelines, using RT has been restricted, the new versions of the National Comprehensive Cancer Network (NCCN) guidelines as well as the National Cancer Center Korea recommended the use of RT as a local treatment modality [3].
It is therefore necessary to have high-quality beam data to avoid dosing errors and patient treatment [1]. Conventional external beam radiation therapy is not usually used for secondary liver cancer; however, two specialized forms of radiotherapy may be used in some cases [2].

In the therapy of hepatocellular carcinoma (HCC), radiotherapy (RT) had a restricted job in the light of the fact that the entire liver is of minimal tolerance to RT notwithstanding the danger of radiation-actuated liver illness (RILD) [2]. Despite the fact that, in numerous rules, utilizing of RT has been limited, the new forms of the National Cancer Center of Korea suggested utilizing of RT as a nearby therapy methodology [3]. There is an obvious association between the dosage of radiation and the possibility of tumor control in several tumors, but the 
tumor dosage is frequently restricted by the radiation tolerance of the surrounding tissues. IMRT may permit saving more normal and healthy tissues as compared to different strategies through more exact adaptation to the selected target. Therefore, this gives the likelihood of both the reduction of late harmfulness and the expansion of the conveyed portion that can bring about progress in tumor control just as endurance [4]. Consequently, this gives the probability of both the decrease of late toxicity and the increase of the delivered dose that can result in improvement in tumor control as well as survival [5].

In HCC, the size of the tumor and its position in the liver determine the appropriate surgery in some patients while radiation therapy is appropriate for others. For example, radiation therapy is a possible treatment for early-stage primary lesions or oligometastatic disease due to its local control of liver diseases [6].

The IMRT has distinct features when compared to the earlier techniques for treatment such as 3DCRT. These features are the converse treatment planning process and the conformal beam which target a large number of therapeutic fields or subfields [7].

Therefore, the IMRT offers an incredible precision and a magnificently conformal portion appropriation through different bars, with a non-uniform force profile for every one of them. There are three sorts of IMRT conveyance frameworks, which permit the development of nonuniform power profiles the moderate multi-leaf collimator (MLC)- mounted straight quickening agents. These three kinds are: (1) step-and-shoot IMRT, where little MLC-produced fragments are utilized, and there is no conveyance for the radiation while the leaves move for the formation of the following section, (2) sliding window IMRT, where tweaked MLC speed in various static radiation fields is utilized, and there is no conveyance for the radiation as the leaves are moving, and (3) volumetric adjusted bend treatment (VMAT), that is a rotational type of IMRT where, all through the turn, moving MLC and changing portion conveyance rates happen [7].

Consequently, the IMRT offers incredible precision and a magnificently conformal dose distribution via multiple beams, with a non-uniform intensity profile for each of them. There are three types of IMRT delivery systems, which allow the formation of non-uniform intensity profiles, equipped with the conservative multileaf collimator (MLC)-mounted linear accelerators. These three types are as follows: (1) step-and-shoot IMRT, where small generated MLC segments are used, and there is no delivery for the radiation while the leaves move for the creation of the next segment, (2) sliding window IMRT, where modulated MLC velocity in multiple static radiation fields are used, and there is no delivery for the radiation as the leaves are moving, and (3) volumetric modulated arc radiation therapy (VMAT), that is a rotational form of IMRT where, throughout the rotation, moving MLC and dynamic dose delivery rates happen [8].

Hence, the principal objective of the examination is to decide the smallness of the objective portion dispersion for liver malignant growth plans utilizing 6MV, 10MV, and DE. Accordingly, evaluation of the conservativeness is conducted regarding tumble off for both higher iso-portion levels and lower isodose levels. This current examination's goal is the measurement of the impact of photon bar energy determination on liver portion dispersions and treatment plan quality.

\section{MATERIALS AND METHODS}

\subsection{Status selection and simulation}

The current retrospective treatment planning study included twenty-eight subjects. The subject was previously treated from liver diseases. Every patient was immobilized by the Body Pro-Lok ${ }^{\mathrm{TM}}$ system (CIVCO, Orange City, IA) with his arms upwards, with application of abdominal compression through a compression plate to reduce the movement of breathing. A free-breathing helical CT scan was applied for all the patients for simulation by a $2 \mathrm{~mm}$ slice thickness. The gross tumor volume (GTV) was segmented where the simulation CT scan study was used by a highly certified radiation oncologist.

Delineation of the planning target volume (PTV) was performed via adding a setup margin, usually $5 \mathrm{~mm}$ in the anterior-posterior and lateral direction and $10 \mathrm{~mm}$ in the superior-inferior direction. The dose prescription and fractionation differed across the patients' sample and ranged from $54 \mathrm{~Gy}$ ( 3 fractions) to $50 \mathrm{~Gy}$ (5 fractions).

\subsection{Beam Energy Selection}

Planning of the initial treatment was done using $6 \mathrm{MV}$ photon energy for all beams. After that, re-planning of the patients was carried out using $10 \mathrm{MV}$ photon energy for all beams. A simple approach based on the central axis depth to the Isocenter was utilized for the dual-energy (DE) photon plans, for a given beam it was used for determination of the used $6 \mathrm{MV}$ or $10 \mathrm{MV}$ energy. Along the beam's central axis to the Isocenter, the effective depth was detected for every patient. For a given patient, all beams' effective depths were 
averaged and the beams possessing an effective depth below the average were set at the energy of $6 \mathrm{MV}$, while the rest were set at 10MV energy. This approach was used to confirm an even split between $6 \mathrm{MV}$ and $10 \mathrm{MV}$ photon energies for the greater part of the patients. The use of 10MV for the greater part was owing to the basis for effective depth that mainly based on that the penetrative power of $10 \mathrm{MV}$ is greater than $6 \mathrm{MV}$.

The total number of the patients was planned in the Monaco treatment planning system (TPS) version 5.112 IMRT beams with $6 \mathrm{MV}, 10 \mathrm{MV}$, and DE. The orientations of the beam were selected for minimizing the beam overlap and OAR irradiation. For plan optimization and dose calculation, DMPO optimization was employed with a final dose calculation by the Monte Carlo, adaptive convolve algorithm calculation for high-quality dependable treatment planning result.

For each patient, there were no variations in the collimator, gantry, and couch angles within a patient when comparing $6 \mathrm{MV}, 10 \mathrm{MV}$, and DE plans. All other settings of the plan were equivalent but differed among patients. All plans were optimized with similar planning objectives and normalized such that $98 \%$ of the PTV received $100 \%$ of the prescription.

\subsection{Treatment planning techniques}

In the present study, the inverse plan Dose Volume Optimizer (DVO version 5.11.02) of the Monaco planning system was used for IMRT technique planning. Within the IMRT technique, the delivery of the radiation dose was done for planning target volume (PTV). For PTV and the other critical organs (as bladder, rectum, lungs, and kidneys), proper dose-volume constraints for IMRT plan optimization were used. For PTV optimization, constraints were such that $100 \%$ PTV volume ought to be $99.2 \%$ and $98 \%$ minimal dose, while maximal dose ought to be less than $102.2 \%$ and $103 \%$ for zero \% volume respectively. Radiation dose deliveries were planned in two phases. IMRT plans were generated for both 6MV photon beam using two delivery modes (SS) and (SW) with seven coplanar non-opposed beam arrangements of $0^{\circ}, 51^{\circ}, 103^{\circ}, 154^{\circ}, 206^{\circ}, 257^{\circ}, 308^{\circ}$ gantry angles for all patients to ensure identical beam angle arrangements. The radiation dose of $50.4 \mathrm{~Gy}$ and $30.6 \mathrm{~Gy}$ with $1.8 \mathrm{~Gy} /$ fraction were planned for the doses to the OARs were restricted by the RTOG guidelines for critical structure dose. Depending on the list of PTV and OAR plan constraints is shown in Tables 1 (Fig. 1), comparative analysis, Dose-volumetric analysis of each energy IMRT plan was performed by both qualitative and quantitative methods for the normal tissue doses.
Table (1): The diagnosing, prescription dose, patient volume and PTV volume for the investigated cases

\begin{tabular}{|c|c|c|c|}
\hline $\begin{array}{c}\text { patient } \\
\text { number }\end{array}$ & $\begin{array}{c}\text { Prescribed } \\
\text { Dose } \\
(\mathbf{c G y})\end{array}$ & $\begin{array}{c}\text { PTV } \\
\text { Volume } \\
(\mathbf{C C})\end{array}$ & $\begin{array}{c}\text { Patient } \\
\text { Volume }\end{array}$ \\
\hline $\mathbf{c c})$
\end{tabular}




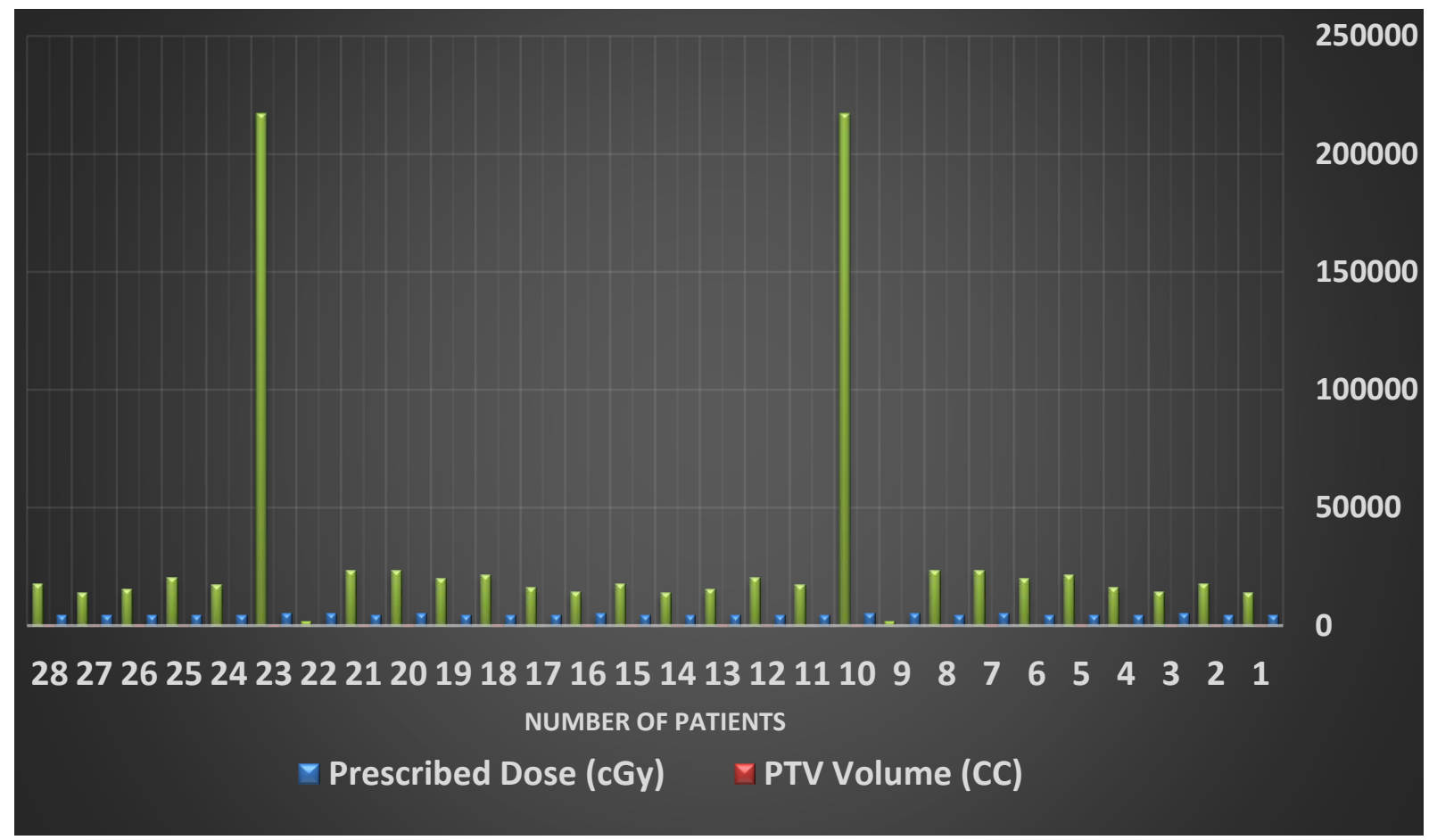

Fig. (1): The diagnosing, prescription dose, patient volume and PTV volume for the investigated cases

For evaluation of the target coverage, it was done in accordance to compare maximum and mean doses to PTV as well as numerous physical indices that were calculated such as ([D $\left.\mathrm{D}_{98 \%(\mathrm{cGy})}\right],\left[\mathrm{D}_{95 \%(\mathrm{cGy})}\right],\left[\mathrm{D}_{5 \%}\right.$ (cGy) $]$, $\left[\mathrm{V}_{95 \%(\%)}\right]$ and $\left[\mathrm{V}_{107 \%(\%)}\right]$. Where $\mathrm{D}_{\min }$ is the minimum dose delivered by percentage value of the PTV. Within PTV, evaluation of the dose homogeneity was done by using Homogeneity Index (HI) as identified by:

$$
H I=D_{5 \%} / D_{95 \%}
$$

Where $\mathrm{D} 95 \%$ and D5\% indicated the dose levels on the curve Dose-Volume Histogram (DVH) which are corresponding to $95 \%$ and $5 \%$ of the target volume, respectively. The HI values, which are near to unity, are indication for higher homogeneity.

For organ at risk, the dose-volume parameters were analyzed for each plan at $6 \mathrm{MV}, 10 \mathrm{MV}$ and $\mathrm{DE}$ by comparing several physical indices. For right lung and left lung, irradiated volumes receiving more than 5, 10, 20, and 30 Gy ( $\mathrm{V}_{5}$ Gy, $\mathrm{V}_{10}$ Gy, $\mathrm{V}_{20}$ Gy and $\mathrm{V}_{30}$ Gy $)$ also $\mathrm{D}_{\text {mean }}, \mathrm{D}_{1 \%}$ and $\mathrm{D}_{5 \%}$ of the lung were calculated. In the remaining cases, the mean doses were calculated [9] for liver and kidney.

\section{RESULTS}

\subsection{The Target Homogeneity and Conformity}

Figure (2) shows the DVH's for 6-MV ,10-MV and DE treatment plans for the liver of some investigated cases. The results of $6 \mathrm{MV}$ plans were shown in a form of large dashed lines (liver IMRT1), the results of 10 MV plans were shown as solid lines(liver IMRT $10 \mathrm{mv}$. and the results of DE MV plans were shown as small dash lines within the majority of the cases, each energy plans has given a similar PTV coverage. Tables (2 and 3) showthe target coverage parameters at 6,10 MV and DE. Figures (3) shows the homogeneity index and the dose-volume parameters of PTV such as D $98 \%$, $\mathrm{D} 95 \%$ and $\mathrm{D} 5 \%$. The quantitative analysis of the results revealed that there were no obvious variations in homogeneity index (HI) among $6 \mathrm{MV}, 10$ Photon beams (average $1.102 \pm 0.011,1.113 \pm 0.015, \mathrm{p}<0.532$ ). Most dose volume indices of PTV are slightly better for 6-MV treatment than 10-MV and it was statistically significant at D5\% ( $\mathrm{p}$ < 0.027). Such a small difference indicates that the lower entrance dose from the high-energy's beam is recovered by the high exit dose. The results showed no differences on the conformity of target between 6-MV treatment plans and the 10-MVplans. 


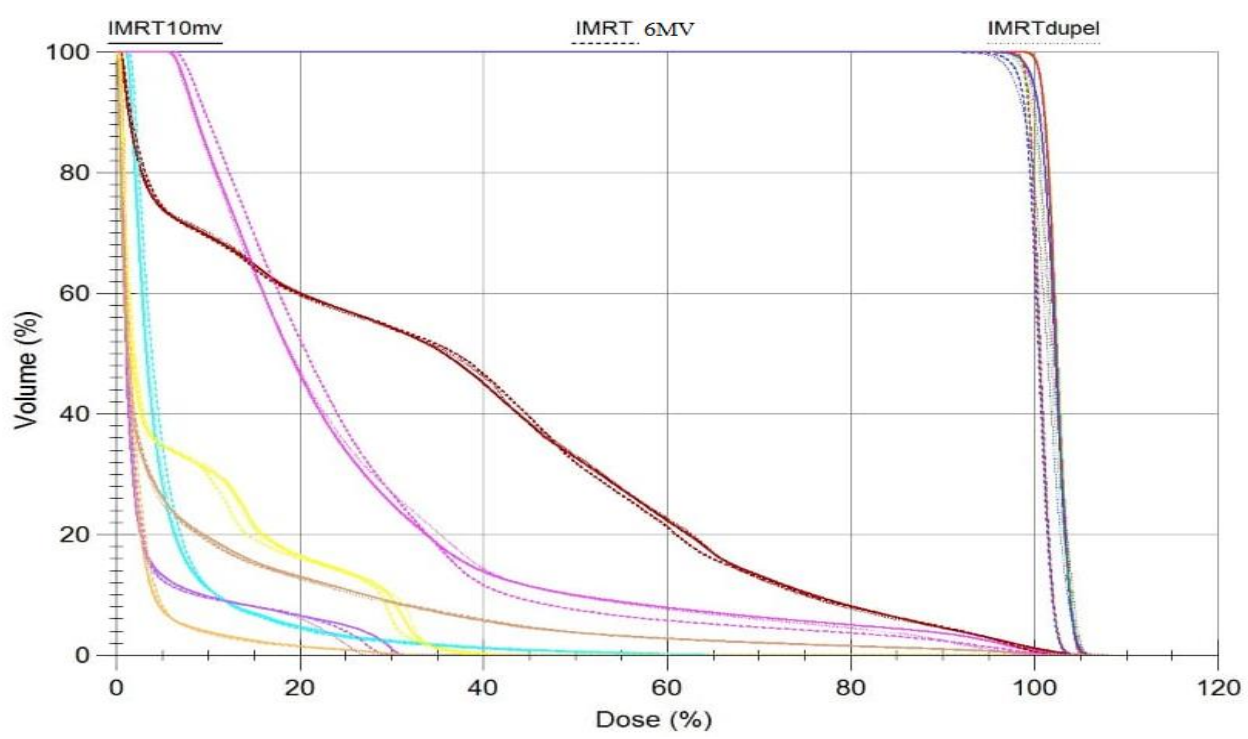

Fig. (2) : the DVH's for of the $6 \mathrm{MV}, 10 \mathrm{MV}$ and DE energies for some treatment plans of the investigated cases

Table (2): Show the mean dose and maximum dose ( $\left.D_{\max }\right)$ to the PTVs for the photon beams $6 \mathrm{MV}, 10 \mathrm{MV}$ and duel energy plans

\begin{tabular}{|c|c|c|c|c|c|c|}
\hline \multirow[t]{2}{*}{ Patient } & \multicolumn{3}{|c|}{ D mean (cGy) } & \multicolumn{3}{|c|}{ D Max (cGy) } \\
\hline & 6 & 10 & 6,10 & 6 & 10 & 6,10 \\
\hline 1 & 4519.4 & 4599.2 & 4555.8 & 4758 & 4833.8 & 4865.2 \\
\hline 2 & 4621.7 & 45821.4 & 4593.8 & 4871 & 4918.2 & 4854.7 \\
\hline 3 & 5527.9 & 5537 & 5533.8 & 5957 & 5880 & 5881.4 \\
\hline 4 & 4567.5 & 4587.9 & 4610.5 & 5106.4 & 4992.8 & 5061.4 \\
\hline 5 & 4592.4 & 4623.7 & 4602.7 & 4862.2 & 4933.9 & 4827.6 \\
\hline 6 & 4596.8 & 4649.1 & 4646.2 & 4967.3 & 5012.3 & 5007.4 \\
\hline 7 & 5427 & 5597.9 & 5579.3 & 5708.1 & 5895.3 & 5853.7 \\
\hline 8 & 4607 & 4636.7 & 4540.8 & 4860 & 4840.8 & 4747.7 \\
\hline 9 & 5482.1 & 5489.3 & 5452.6 & 5724 & 5963.2 & 5982.3 \\
\hline 10 & 5452.3 & 5483.1 & 55142 & 5914.4 & 5895.1 & 5889.5 \\
\hline 11 & 4523.3 & 4543.1 & 4512.4 & 4878.4 & 4891.3 & 4925.2 \\
\hline 12 & 4668.2 & 4730.2 & 4550.2 & 5027.3 & 5187 & 4954.9 \\
\hline 13 & 4519.4 & 4599.2 & 4555.8 & 4758 & 4833.8 & 4865.2 \\
\hline 14 & 4621.7 & 45821.4 & 4593.8 & 4871 & 4918.2 & 4854.7 \\
\hline 15 & 5527.9 & 5537 & 5533.8 & 5957 & 5880 & 5881.4 \\
\hline 16 & 4567.5 & 4587.9 & 4610.5 & 5106.4 & 4992.8 & 5061.4 \\
\hline 17 & 4592.4 & 4623.7 & 4602.7 & 4862.2 & 4933.9 & 4827.6 \\
\hline 18 & 4596.8 & 4649.1 & 4646.2 & 4967.3 & 5012.3 & 5007.4 \\
\hline 19 & 5427 & 5597.9 & 5579.3 & 5708.1 & 5895.3 & 5853.7 \\
\hline 20 & 4607 & 4636.7 & 4540.8 & 4860 & 4840.8 & 4747.7 \\
\hline 21 & 5482.1 & 5489.3 & 5452.6 & 5724 & 5963.2 & 5982.3 \\
\hline 22 & 5452.3 & 5483.1 & 55142 & 5914.4 & 5895.1 & $\mathbf{5 8 8 9 . 5}$ \\
\hline 23 & 4523.3 & 4543.1 & 4512.4 & 4878.4 & 4891.3 & 4925.2 \\
\hline 24 & 4668.2 & 4730.2 & 4550.2 & 5027.3 & 5187 & 4954.9 \\
\hline 25 & 4519.4 & 4599.2 & 4555.8 & 4758 & 4833.8 & 4865.2 \\
\hline 26 & 4621.7 & 45821.4 & 4593.8 & 4871 & 4918.2 & 4854.7 \\
\hline 27 & 5527.9 & 5537 & 5533.8 & 5957 & 5880 & 5881.4 \\
\hline 28 & 4567.5 & 4587.9 & 4610.5 & 5106.4 & 4992.8 & 5061.4 \\
\hline Mean & 4871.70 & 9326.53 & 8426.22 & 5212.88 & 5254.01 & 5227.31 \\
\hline SD & 427.66 & 12881.09 & 13200.86 & 454.28 & 464,84 & 478.62 \\
\hline P Value & \multicolumn{3}{|c|}{0.260106} & \multicolumn{3}{|c|}{0.949095} \\
\hline
\end{tabular}




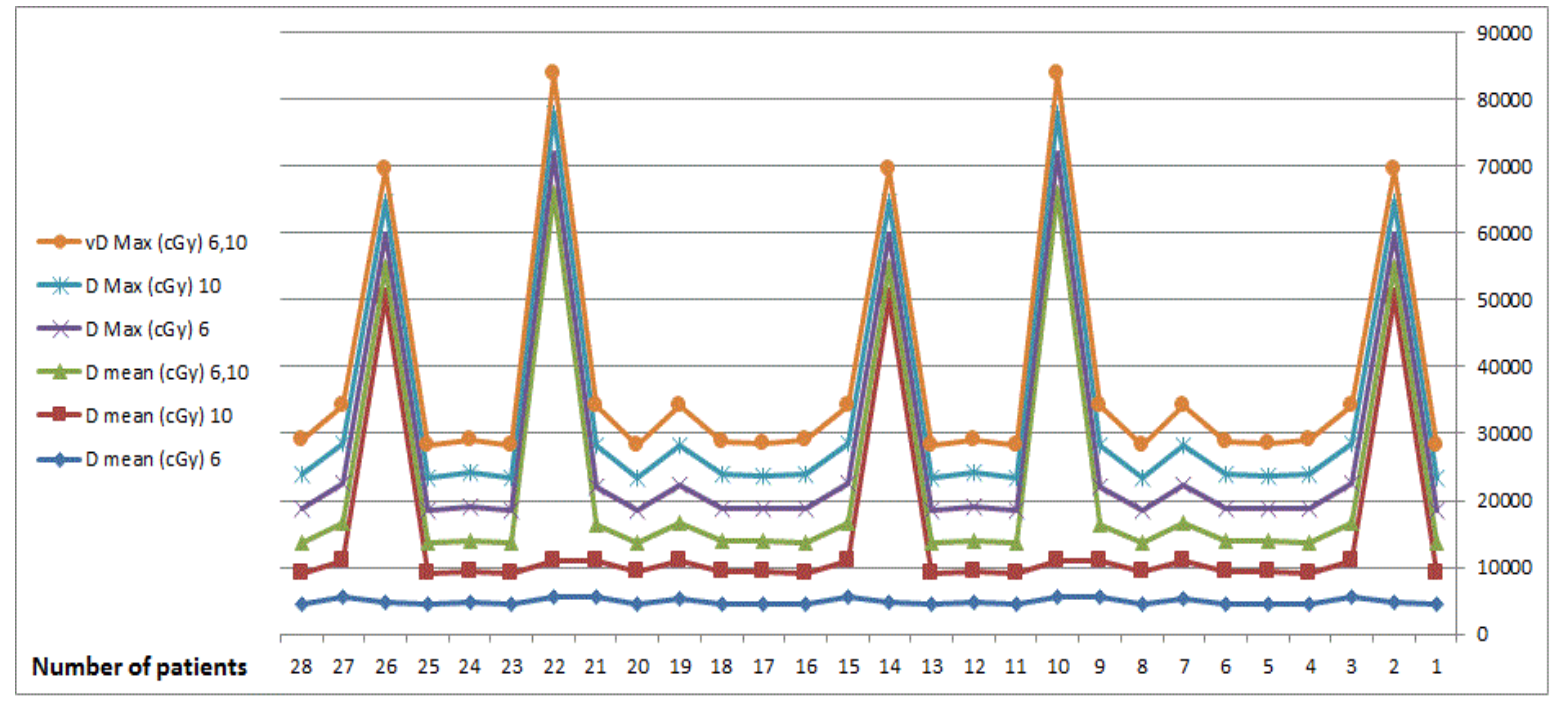

Fig. (3): The mean dose and maximum dose

Table (3): Show the volume received $95 \%$ and $107 \%$ of dose $\left(V_{95}, V_{107 \%}\right)$ to the PTVs for the $6 \mathrm{MV}, 10 \mathrm{MV}$ and DE plans

\begin{tabular}{|c|c|c|c|c|c|c|}
\hline \multirow{2}{*}{ Patient } & \multicolumn{3}{|c|}{$\mathrm{V}_{95 \%}(\%)$} & \multicolumn{3}{|c|}{$\mathrm{V}_{107 \%}(\%)$} \\
\hline & 6 & 10 & 6,10 & 6 & 10 & 6,10 \\
\hline 1 & 98.22 & 99.54 & 98.15 & 0.22 & 0.33 & 0.09 \\
\hline 2 & 99.69 & 99.97 & 99.46 & 0 & 0 & $\mathbf{0}$ \\
\hline 3 & 99.71 & 98.95 & 99.36 & 0.02 & 0.02 & 0.01 \\
\hline 4 & 95.79 & 96.86 & 97.06 & 2.83 & 2.56 & 3.9 \\
\hline 5 & 99.69 & 99.87 & 99.86 & 0 & 0.02 & $\mathbf{0}$ \\
\hline 6 & 99.62 & 98.68 & 98.71 & 0.85 & 3.21 & 3.09 \\
\hline 7 & 99.99 & 100 & 100 & 0 & 0.09 & 0.08 \\
\hline 8 & 99.53 & 100 & 99.94 & 0 & 0.01 & $\mathbf{O}$ \\
\hline 9 & 97.12 & 95.98 & 92.80 & 7.66 & 3.721 & 0.34 \\
\hline 10 & 98.22 & 99.54 & 98.15 & 0.22 & 0.33 & 0.09 \\
\hline 11 & 99.69 & 99.97 & 99.46 & 0 & 0 & $\mathbf{0}$ \\
\hline 12 & 99.71 & 98.95 & 99.36 & 0.02 & 0.02 & 0.01 \\
\hline 13 & 95.79 & 96.86 & 97.06 & 2.83 & 2.56 & 3.9 \\
\hline 14 & 99.69 & 99.87 & 99.86 & 0 & 0.02 & $\mathbf{0}$ \\
\hline 15 & 99.62 & 98.68 & 98.71 & 0.85 & 3.21 & 3.09 \\
\hline 16 & 99.99 & 100 & 100 & 0 & 0.09 & 0.08 \\
\hline 17 & 99.53 & 100 & 99.94 & 0 & 0.01 & $\mathbf{O}$ \\
\hline 18 & 97.12 & 95.98 & 92.80 & 7.66 & 3.721 & 0.34 \\
\hline 19 & 98.22 & 99.54 & 98.15 & 0.22 & 0.33 & 0.09 \\
\hline 20 & 99.69 & 99.97 & 99.46 & 0 & 0 & $\mathbf{0}$ \\
\hline 21 & 99.71 & 98.95 & 99.36 & 0.02 & 0.02 & 0.01 \\
\hline 22 & 95.79 & 96.86 & 97.06 & 2.83 & 2.56 & 3.9 \\
\hline 23 & 99.69 & 99.87 & 99.86 & 0 & 0.02 & $\mathbf{0}$ \\
\hline 24 & 99.62 & 98.68 & 98.71 & 0.85 & 3.21 & 3.09 \\
\hline 25 & 99.99 & 100 & 100 & 0 & 0.09 & 0.08 \\
\hline 26 & 99.53 & 100 & 99.94 & 0 & 0.01 & $\mathbf{O}$ \\
\hline 27 & 97.12 & 95.98 & 92.80 & 7.66 & 3.721 & 0.34 \\
\hline 28 & 98.22 & 99.54 & 98.15 & 0.22 & 0.33 & 0.09 \\
\hline Mean & 98.80 & 98.90 & 98.36 & 1.25 & 1.08 & 0.90 \\
\hline SD & 1.39 & 1.41 & 2.17 & 2.42 & 1.49 & 1.50 \\
\hline P Value & & .50638 & & & .66675 & \\
\hline
\end{tabular}




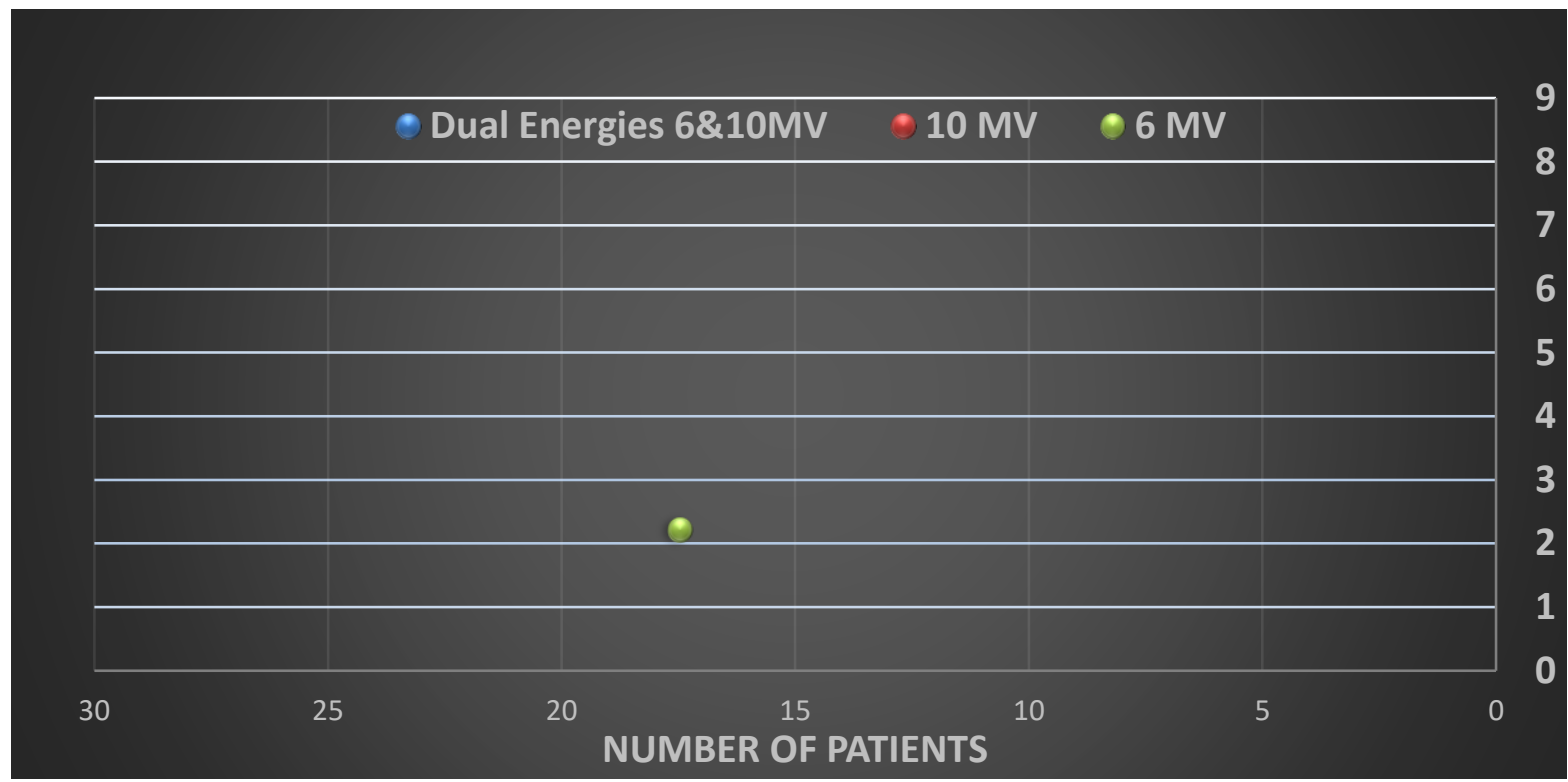

Fig. (4): The volume received $107 \%$ to the PTVs for the $6 \mathrm{MV}, 10 \mathrm{MV}$ and DE plans

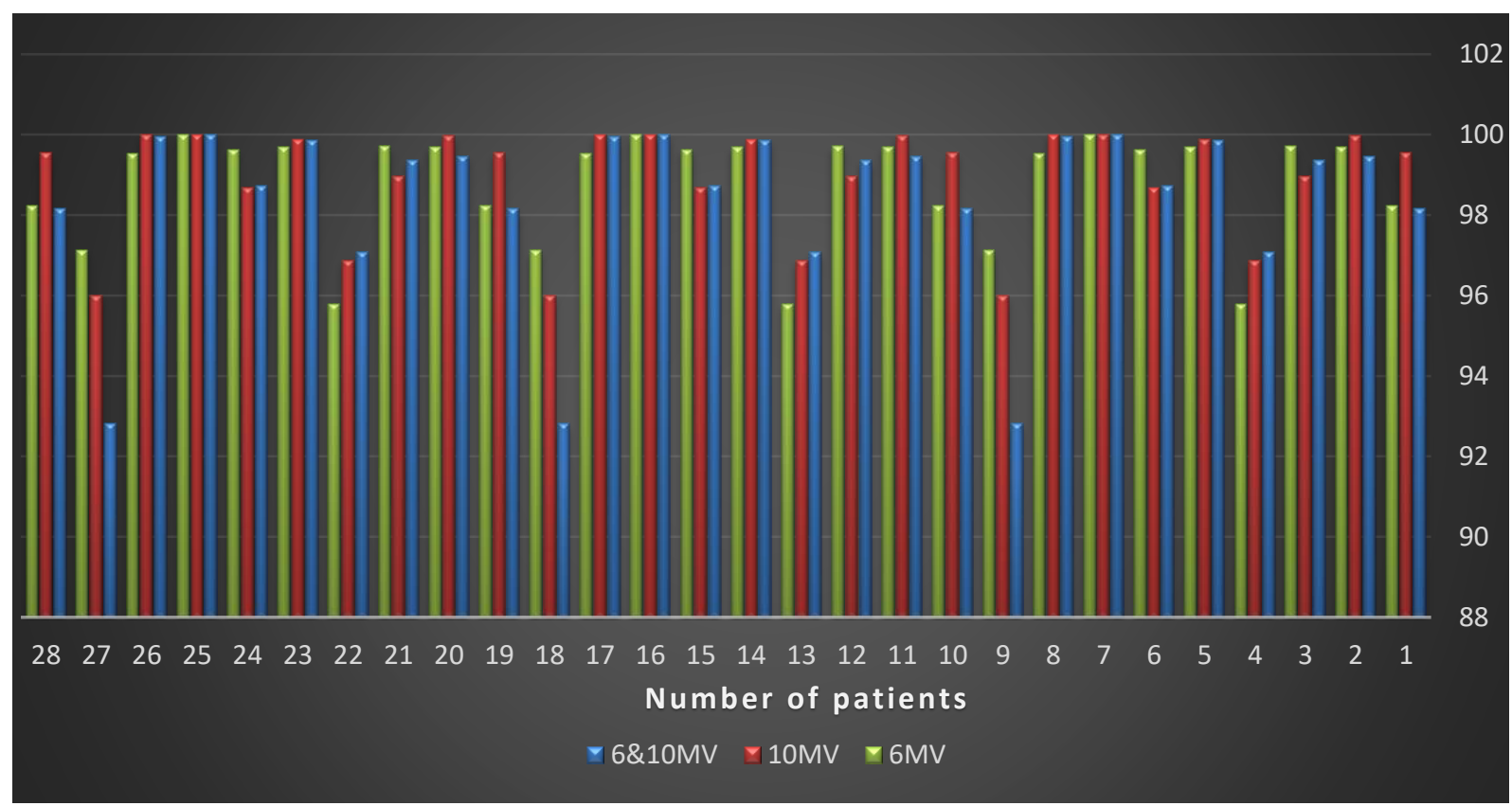

Fig. (5): The volume received 95\% to the PTVs for the 6MV, 10MV and DE plans

\subsection{Doses for different organs}

\section{Dose to Lung, Kidney and Liver}

In both lungs, 10-MV plans gave better sparing of in various dose volume parameters however, there were no statistically significant difference in the results except in D5\% of left lung $(p<0.01)$. In addition, DVHs for kidneys and liver exhibit the same behavior of the lung. $6 \mathrm{MV}$ and $10 \mathrm{MV}$ plans provided the mean doses of liver and kidneys below the tolerance limits.

\section{Dose for Normal Tissue}

The body volumes D2\% and D5\% doses received a slightly lower dose with 10-MV than 6-MV photon beams. For D5\%, the change was statistically significant $(\mathrm{p}<0.05426)$ (Table 5). For each patient, the receiving $\mathrm{V}_{2 \%} \mathrm{CGy}$ and $\mathrm{V}_{5 \%} \mathrm{CGy}$ were compared. The general tendency was that $10 \mathrm{MV}$ treatment plans showed the lowest volume received in more than 2 and $5 \mathrm{~Gy}$, and 6 MV beams treatment plans showed the highest volume. 
Table (4): the dose-volume parameters for the different OAR according to the different cases at all energies

\begin{tabular}{|c|c|c|c|c|}
\hline OAR & DVH parameters & $10 \mathrm{MV}$ & $6 \mathrm{MV}$ & P value \\
\hline \multirow{6}{*}{ RT lung } & V5Gy (\%) & $62.46 \pm 31.04$ & $62.95 \pm 30.81$ & 0.189 \\
\hline & V10Gy (\%) & $48.3 \pm 33.73$ & $47.63 \pm 34.42$ & 0.22 \\
\hline & V20Gy (\%) & $26.18 \pm 26.24$ & $26.46 \pm 26.55$ & 0.47 \\
\hline & V30Gy (\%) & $18.17 \pm 23.16$ & $18.26 \pm 20.28$ & 0.17 \\
\hline & D1\% (Gy) & $37.82 \pm 23.31$ & $37.82 \pm 21.84$ & 0.86 \\
\hline & D5\% (Gy) & $35.71 \pm 24.67$ & $35.69 \pm 25.39$ & 0.64 \\
\hline \multirow{7}{*}{ LT lung } & Mean & $16.98 \pm 8.98$ & $16.87 \pm 9.61$ & 0.25 \\
\hline & V5Gy (\%) & $80.31 \pm 4.98$ & $81 \pm 5.12$ & 0.35 \\
\hline & V10Gy (\%) & $56.98 \pm 21.10$ & $56.99 \pm 20.21$ & 0.41 \\
\hline & V20Gy (\%) & $30 \pm 23.88$ & $30.89 \pm 24.11$ & 0.32 \\
\hline & V30Gy (\%) & $18.89 \pm 21.42$ & $18.23 \pm 21.51$ & 0.17 \\
\hline & D1\% (Gy) & $43.3 \pm 17.52$ & $43.43 \pm 17.51$ & 0.54 \\
\hline & D5\% (Gy) & $40.1 \pm 18.76$ & $40.46 \pm 18.75$ & 0.01 \\
\hline RT kidney & Mean (Gy) & $9.52 \pm 4.5$ & $9.62 \pm 4.64$ & 0.04 \\
\hline LT kidney & Mean (Gy) & $8.4 \pm 2.98$ & $8.5 \pm 2.84$ & 0.001 \\
\hline Liver & Mean (Gy) & $6.03 \pm 2.2$ & $6.21 \pm 2.11$ & 0.22 \\
\hline
\end{tabular}

Table (5): A comparison low dose distribution in healthy tissue

\begin{tabular}{|c|c|c|c|c|c|c|c|c|c|c|c|c|}
\hline \multirow{2}{*}{$\begin{array}{c}\text { Patient } \\
\text { no }\end{array}$} & \multicolumn{3}{|c|}{ D2\% (CGy) } & \multicolumn{3}{|c|}{ D5\%(CGy) } & \multicolumn{3}{|c|}{ V2\%CGy } & \multicolumn{3}{|c|}{ V5\%CGy } \\
\hline & 6 & 10 & $6+10$ & 6 & 10 & $6+10$ & 6 & 10 & $6+10$ & 6 & 10 & $6+10$ \\
\hline 1 & 2924.6 & 2849.7 & 2948 & 1992.8 & 1927.6 & 1948.3 & 19.56 & 18.91 & 19.15 & 13.26 & 13.88 & 13.41 \\
\hline 2 & 3121.8 & 3204.7 & 3154.1 & 1955.9 & 1923.4 & 1901.7 & 27.89 & 27.62 & 26.46 & 17.82 & 18.53 & 18.09 \\
\hline 3 & 2202.7 & 2168.4 & 218.4 & 1635.6 & 1607.8 & 1603.7 & 25.75 & 25.72 & 25.39 & 18.17 & 18.66 & 18.39 \\
\hline 4 & 3045.9 & 2917.6 & 2980.6 & 1347 & 1387.5 & 1393.1 & 23.85 & 2392 & 23.99 & 14.03 & 14.99 & 14.72 \\
\hline 5 & 1758 & 1678.2 & 1731.9 & 1417.2 & 1188.4 & 1223.4 & 19.80 & 19.39 & 19.35 & 11.81 & 12.39 & 11.68 \\
\hline 6 & 2776 & 3202.4 & 3229.1 & 2035.8 & 2268.9 & 23278 & 36.77 & 36.27 & 36.97 & 27.02 & 25.29 & 25.34 \\
\hline 7 & 2546.7 & 2550.1 & 2480.8 & 1852.7 & 1773.1 & 1857.6 & 22.24 & 22.01 & 21.99 & 16.63 & 17.45 & 16.65 \\
\hline 8 & 1786.3 & 1738.4 & 1802.9 & 858.3 & 1009.1 & 971.4 & 11.13 & 11.80 & 11.72 & 6.88 & 7.94 & 7.22 \\
\hline 9 & 3137.5 & 3036.6 & 2930.4 & 2429.8 & 2417.2 & 2328.1 & 28.8 & 27.85 & 27.89 & 20.47 & 20.77 & 19.96 \\
\hline 10 & 2924.6 & 2849.7 & 2948 & 1992.8 & 1927.6 & 1948.3 & 19.56 & 18.91 & 19.15 & 13.26 & 13.88 & 13.41 \\
\hline 11 & 3121.8 & 3204.7 & 3154.1 & 1955.9 & 1923.4 & 1901.7 & 27.89 & 27.62 & 26.46 & 17.82 & 18.53 & 18.09 \\
\hline 12 & 2202.7 & 2168.4 & 218.4 & 1635.6 & 1607.8 & 1603.7 & 25.75 & 25.72 & 25.39 & 18.17 & 18.66 & 18.39 \\
\hline 13 & 3045.9 & 2917.6 & 2980.6 & 1347 & 1387.5 & 1393.1 & 23.85 & 2392 & 23.99 & 14.03 & 14.99 & 14.72 \\
\hline 14 & 1758 & 1678.2 & 1731.9 & 1417.2 & 1188.4 & 1223.4 & 19.80 & 19.39 & 19.35 & 11.81 & 12.39 & 11.68 \\
\hline 15 & 2776 & 3202.4 & 3229.1 & 2035.8 & 2268.9 & 23278 & 36.77 & 36.27 & 36.97 & 27.02 & 25.29 & 25.34 \\
\hline 16 & 2546.7 & 2550.1 & 2480.8 & 1852.7 & 1773.1 & 1857.6 & 22.24 & 22.01 & 21.99 & 16.63 & 17.45 & 16.65 \\
\hline 17 & 1786.3 & 1738.4 & 1802.9 & 858.3 & 1009.1 & 971.4 & 11.13 & 11.80 & 11.72 & 6.88 & 7.94 & 7.22 \\
\hline 18 & 3137.5 & 3036.6 & 2930.4 & 2429.8 & 2417.2 & 2328.1 & 28.8 & 27.85 & 27.89 & 20.47 & 20.77 & 19.96 \\
\hline 19 & 2924.6 & 2849.7 & 2948 & 1992.8 & 1927.6 & 1948.3 & 19.56 & 18.91 & 19.15 & 13.26 & 13.88 & 13.41 \\
\hline 20 & 3121.8 & 3204.7 & 3154.1 & 1955.9 & 1923.4 & 1901.7 & 27.89 & 27.62 & 26.46 & 17.82 & 18.53 & 18.09 \\
\hline 21 & 2202.7 & 2168.4 & 218.4 & 1635.6 & 1607.8 & 1603.7 & 25.75 & 25.72 & 25.39 & 18.17 & 18.66 & 18.39 \\
\hline 22 & 3045.9 & 2917.6 & 2980.6 & 1347 & 1387.5 & 1393.1 & 23.85 & 2392 & 23.99 & 14.03 & 14.99 & 14.72 \\
\hline 23 & 2924.6 & 2849.7 & 2948 & 1992.8 & 1927.6 & 1948.3 & 19.56 & 18.91 & 19.15 & 13.26 & 13.88 & 13.41 \\
\hline 24 & 3121.8 & 3204.7 & 3154.1 & 1955.9 & 1923.4 & 1901.7 & 27.89 & 27.62 & 26.46 & 17.82 & 18.53 & 18.09 \\
\hline 25 & 2202.7 & 2168.4 & 218.4 & 1635.6 & 1607.8 & 1603.7 & 25.75 & 25.72 & 25.39 & 18.17 & 18.66 & 18.39 \\
\hline 26 & 3045.9 & 2917.6 & 2980.6 & 1347 & 1387.5 & 1393.1 & 23.85 & 2392 & 23.99 & 14.03 & 14.99 & 14.72 \\
\hline 27 & 1758 & 1678.2 & 1731.9 & 1417.2 & 1188.4 & 1223.4 & 19.80 & 19.39 & 19.35 & 11.81 & 12.39 & 11.68 \\
\hline 28 & 2776 & 3202.4 & 3229.1 & 2035.8 & 2268.9 & 23278 & 36.77 & 36.27 & 36.97 & 27.02 & 25.29 & 25.34 \\
\hline Mean & 2633.0 & 2637.6 & 2375.6 & 1727.4 & 1719.9 & 3971.6 & 24.4 & 362.4 & 24.0 & 16.3 & 16.8 & 16.3 \\
\hline SD & 510.4 & 555.4 & 1028.0 & 394.7 & 411.4 & 6819.8 & 6.3 & 843.8 & 6.2 & 5.1 & 4.5 & 4.6 \\
\hline P Value & \multicolumn{3}{|c|}{0.317987} & \multicolumn{3}{|c|}{0.05426} & \multicolumn{3}{|c|}{0.014048} & \multicolumn{3}{|c|}{0.923365} \\
\hline
\end{tabular}




\section{DISCUSSION}

IMRT is considered one of the major critical advancements in radiotherapy in current years. It can provide the capability of improving clinical outcomes and decrease morbidity. IMRT, indeed, is still developing as a therapeutic modality, but once launched, it is likely to lower the times of planning and treatment.

In the current study, the effect of $6 \mathrm{MV}, 10 \mathrm{MV}$, and DE beams' energy on liver cancer with a higher target dose compactness were evaluated in the IMRT delivery technique.

Comparing all the above parameters, it was shown that there was a slight variation between $6 \mathrm{MV}, 10 \mathrm{MV}$, and DE. Both lungs' $10 \mathrm{MV}$ plans were continually superior on $6 \mathrm{MV}$ plans, but both were clinically equivalent because the lung is a comparatively a large organ. Therefore, while it exhibits a higher partial volume effect, a small increase in dose is unlikely to elevate its complication possibility which is considerably similar and in agreement with previously published data[10].

The role of using different energies in the current study is reflected due to the power of penetration, the irradiated volume of integral dose and the low dose increase in $6 \mathrm{MV}$ plans. Acute or sub-acute clinical morbidity might not be caused by that low-dose volume; however, it might possibly be carcinogenic [11]. Statistically, the results showed significant variations between $6 \mathrm{MV}$ and 10MV for the parameters. In a study on the investigation of the feasibility of $6 \mathrm{MV}$ intensitymodulated photons usage for the treatment of very large prostate cancer patients, by Sun and Ma, it was shown that using the $6 \mathrm{MV}$ is an efficient choice for treating even very large prostate cancer patients [12]

In addition, photon beams of lower energy (6 MV) were more preferable than higher energies $(15-18 \mathrm{MV})$ in treating tumors that adjoin lung tissues [13]. Another study by Gopi solaiappan et al., investigating the effect of beam energy on the IMRT plans quality, with detailed analysis to all the DVH parameters, using $6 \mathrm{MV}$ photons for IMRT of prostate cancer was recommended [14]. Consequently, nearly the total of previous researches revealed that the use of low-energy photon beams in IMRT was more favored than the higher energies.

However, the situation in 10MV usage was different as $10 \mathrm{MV}$ photon beams areon the threshold energies border for the stimulation of lethal secondary cancers.

In a study by Sung $\mathrm{W}$ et al., in which a comparison on the effect of three-photon energies $(6 \mathrm{MV}, 10 \mathrm{MV}$, and
$15 \mathrm{MV}$ ) was conducted on IMRT plans for treatment of twenty patients with prostate cancer, it was found that 10MV plans revealed better OARs sparing and fewer integral doses than $6 \mathrm{MV}$. In concord with that work, the present study suggests that using $10 \mathrm{MV}$ photon beams was diametrical compared with 6MV photon beams in terms of homogeneity, target volume coverage, conformity, and OARs sparing. It was found that the normal tissues surrounding the target volume got higher doses for the 6MV than 10MV beams; however, it must be taken into account that there are no secondary neutrons associated with $6 \mathrm{MV}$, and radiation seepage was somewhat low. In addition, 6MV photons had significant less room shielding requirements than those required for 10MV photons [15]. The uncertainty within the dose given to a patient must be between 3-5\% [16]

\section{CONCLUSION}

It can be concluded that the use of high-energy 10$\mathrm{MV}$ and DE photon in the treatment plan gives the same tumor control achieved by a 6-MV photon with some complications. Using this plan results in complications of an acceptable rate and saves the normal tissue. It is recommended that treatment options at $10 \mathrm{MV}$ and $\mathrm{DE}$ should be considered a risk-versus-benefit strategy, as clinical significance remains to be determined according to the individual cases. the present study suggests the dosimetric benefits of high energies as well as low energy for liver treatment. Moreover, results of the current study could be applied to choose the best plan for HCC to compare OAR doses and select the best for patient treatment with target coverage.

\section{REFERENCES}

[1] S.Hassn , Nashaat A. Deiab, and Arafa H. Aly, " Dosimetric Study of photon Beam characteristics with 2D Array and water phantom Measurement" Int. J. Radiat. Res., Vol. 18(1), 167,2020 .

[2] Nakamura, Katsumasa, et al. Recent advances in radiation oncology: intensity-modulated radiotherapy, a clinical perspective. International journal of clinical oncology, 2014, 19.4: 564-569.

[3] KIM, Jieun; JUNG, Youngmi. Radiation-induced liver disease: current understanding and future perspectives. Experimental \& molecular medicine, 2017, 49.7: e359-e359.

[4] Scorsetti, M., Comito, T., Cozzi, L., Clerici, E., Tozzi, A., Franzese, C., \& Iftode, C. The challenge of inoperable hepatocellular carcinoma (HCC): results of a single-institutional experience on 
stereotactic body radiation therapy (SBRT). Journal of cancer research and clinical oncology, 2015, 141.7: 1301-1309.

[5] Bae, Sun Hyun; JANG, Won IL; PARK, Hee Chul. Intensity-modulated radiotherapy for hepatocellular carcinoma: dosimetric and clinical results. Oncotarget, 2017, 8.35: 59965.

[6] Choi, Seo Hee; SEONG, Jinsil. Strategic application of radiotherapy for hepatocellular carcinoma. Clinical and molecular hepatology, 2018, 24.2: 114 .

[7] Zhang, Haige, et al. Image-guided intensitymodulated radiotherapy improves short-term survival for abdominal lymph node metastases from hepatocellular carcinoma. Annals of Palliative Medicine, 2019, 8.5: 717-727.

[8] Kim, J. W., Han, K. H., \& Seong, J. Phase I/II trial of helical IMRT-based stereotactic body radiotherapy for hepatocellular carcinoma. Digestive and Liver Disease, 2019, 51.3: 445-451.

[9] Benedict, S. Yenice, KM. Followill, D. et al. Stereotactic body radiation therapy: The report of AAPM Task Group 101. Med.Phys. 37(8):40784101;2010.

[10] HAURI, Pascal; SCHNEIDER, Uwe. Whole-body dose equivalent including neutrons is similar for 6 MV and 15 MV IMRT, VMAT, and 3D conformal radiotherapy. Journal of applied clinical medical physics, 2019, 20.3: 56-70.

[11] TAMILARASU, Suresh, et al. Comparative Evaluation of a 6MV Flattened Beam and a Flattening Filter Free Beam for Carcinoma of Cervix-IMRT Planning Study. Asian Pacific journal of cancer prevention: APJCP, 2018, 19.3: 639.

[12] AL-SHAREEF, Jamal, et al. Comparison of intensity modulated and 3-dimensional conformal radiotherapy for prostate cancer using 6-MV and 15-MV photon energies. Arab Journal of Nuclear Sciences and Applications, 2020, 53.2: 189-200.

[13] Eldesoky, Ismail, Ehab M. Attalla, and Wael M. Elshemey. "The Dosimetric Effects of Different Beam Energy on Physical Dose Distributions in IMRT Based on Analysis of Physical Indices." Journal of Cancer Therapy 4.11, 2013, 33.

[14] G. Solaiappan, G. Singaravelu, A. Prakasarao, B. Rabbani and S. S. Supe, "Influence of Photon Beam Energy on IMRT Plan Quality for Radiotherapy of Prostate Cancer," Reports of Practical Oncology and Radiotherapy, Vol. 14, No. 1, 2009, pp. 18-31.

[15] W. Sung, J. M. Park, C. H. Choi, S. W. Ha and S. J. Ye, "The Effect of Photon Energy on IntensityModulated Ra- diation Therapy (IMRT) Plans for Prostate Cancer," Jour- nal of Radiation Oncology, Vol. 30, No. 1, 2012, pp. 27- 35.

[16] S. Hassn, Nashaat A. Deiab, and Arafa H. Aly " Comparative Study and Dose Evaluation of Photon Beam for water phantom, 2D-array and Treatment Planning System in Small Field sizes" Arab journal of nuclear sciences and applications, 2020,53,1,119-124. 